\title{
Molecular Motions of $\alpha$-Cyclodextrin on a Dodecyl Chain Studied by Molecular Dynamics Simulations
}

\author{
Seiji Ishikawa, ${ }^{a}$ Shun Hirota, ${ }^{a, b}$ Saburo Neya, ${ }^{c}$ and Noriaki Funasaki ${ }^{*, a}$ \\ ${ }^{a}$ Department of Physical Chemistry, 21st Century COE Program, Kyoto Pharmaceutical University; Misasagi, Yamashina- \\ ku, Kyoto 607-8414, Japan: ${ }^{b}$ PRESTO, Japan Science and Technology Agency; Kawaguchi, Saitama 332-0012, Japan: \\ and ${ }^{c}$ Graduate School of Pharmaceutical Sciences, Chiba University; Inage-Yayoi, Chiba 263-8522, Japan. \\ Received November 30, 2005; accepted January 18, 2006; published online January 24, 2006
}

\begin{abstract}
Motions of an $\alpha$-cyclodextrin $(\alpha-\mathrm{CD})$ molecule on a dodecyl chain adopting the all-trans conformation were investigated in the presence of water by molecular dynamics simulations with CVFF force fields, where the trimethylammonium group of dodecyltrimethylammonium bromide (DTAB) is protruded outside the secondary hydroxyl rim of $\alpha$-CD (the secondary-in structure). The $\alpha$-CD molecule shuttled rapidly on the chain without decomplexation. This rapid motion is consistent with the NMR data. The plane formed by 604 atoms of $\alpha$-CD is most populated between the $\mathrm{C} 6$ and $\mathrm{C} 7$ atoms of $\mathrm{DTAB}$. This structure is very close to that estimated by NMR. The $\alpha$-CD molecule underwent a restricted rotation in a range of $60^{\circ}$ with regard to the plane of the dodecyl chain: this plane at the most population is middle between the two diagonal lines of the normal hexagon formed by 604 atoms of $\alpha$-CD. The published NMR data were reanalyzed in terms of the rotation angle, and a slightly better structure was obtained. The distortion of the $\alpha$-CD cavity from the normal hexagon was decreased upon complex formation with DTAB. The deviation of the center of $\alpha$-CD from the center of the dodecyl chain predicted by molecular dynamics simulations is consistent with the NMR data. The secondary-in structure is energetically more stable than the primary-in structure, as calculated by molecular mechanics with CVFF and Amber force fields. This result is consistent with the NMR data. Molecular dynamics simulations were also carried out for the primary-in structure. Some of the results are close to those of the secondary-in structure.
\end{abstract}

Key words molecular dynamics; $\alpha$-cyclodextrin; surfactant; NMR; complex

Cycloamyloses (CD) are doughnut-shaped molecules, formed from $\mathrm{D}(+)$-glucose units linked in a cycle. The interior of the doughnut predominantly contains $\mathrm{CH}$ groups. It provides, therefore, a relatively hydrophobic environment into which nonpolar molecules can be trapped. This characteristic allows CD to bind a variety of smaller guest molecules, leading to academic studies and industrial applications. The academic studies include intermolecular interactions, molecular recognition, chiral separations, and enzymatic catalysis models. ${ }^{1-3)}$

The structures of $\mathrm{CD}$ complexes are determined by X-ray analysis, ${ }^{4,5)}$ NMR, ${ }^{6)}$ and circular dichroism. ${ }^{1,2)}$ Despite these intensive experimental studies, the characteristics of the $\mathrm{CD}$ complexes with smaller molecules are only partially understood at the atomic level. Computational techniques, such as calculations by molecular mechanics, molecular dynamics, molecular orbital method, and molecular surface areas, are providing to be valuable tools for the isolations and understanding of the factors that determine the strength and geometry of binding. In particular, molecular dynamics simulations can predict molecular vibrations, rotation about conformationally mobile bonds, and translations through space, although these predictions have not yet been compared with experiments. $^{7,8)}$

The cavity of cyclohexaamylose $(\alpha-\mathrm{CD})$ has an inner diameter of approximately $0.45 \mathrm{~nm}$, as shown in Fig. 1. This CD cavity, therefore, can accommodate surfactants very well. Complex formation between surfactants and $\mathrm{CD}$ has been extensively investigated by electrochemical, ${ }^{9,10)}$ surface chemical, ${ }^{11,12)} \mathrm{NMR},{ }^{13-18)}$ crystallographic, ${ }^{19,20)}$ and computational methods. ${ }^{21-23)}$ The solution structures of complexes of $\mathrm{CD}$ with surfactants and related compounds are estimated from ROESY spectra and chemical shifts and compared with molecular mechanics and molecular surface area calculations. ${ }^{6-8,14-17,21-24)}$ Recently, on the basis of intensity data of intermolecular ROESY cross-peaks, we determined rather detailed solution structures of $\alpha$-CD complexes with propanol, ${ }^{24)}$ hexyltrimethylammonium, octyltrimethylammonium, ${ }^{16)}$ and dodecyltrimethylammonium (DTAB) bromides. ${ }^{17)}$ It was suggested that an $\alpha$-CD molecule complexed with a surfactant molecule moves more extensively on the alkyl chain, as the alkyl chain becomes longer. ${ }^{16,17)}$ The effect of protonation and chain length on complexation between $\alpha$ $\mathrm{CD}$ and $\alpha, \omega$-diaminoalkanes was investigated by NMR diffusion measurements. ${ }^{15)}$ To our knowledge, no molecular dy-

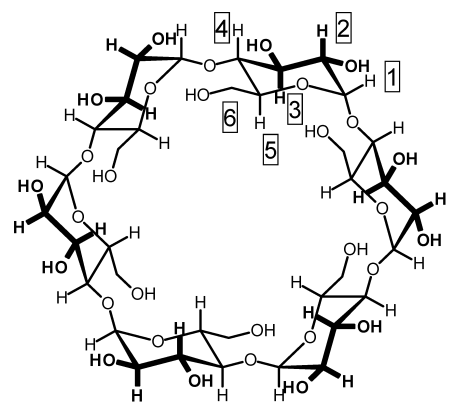

$\alpha-C D$<smiles>CCCCCCCCCCCCCCCCCCCCCCCCC</smiles>

DTAB

Fig. 1. Chemical Structures of DTAB and $\alpha$-CD and Labeling of Their Proton Atoms

An $\alpha$-CD molecule consists of six glucose units connected by six $\alpha-1-4$ linkages. Each glucose unit contains six carbon atoms $(\mathrm{C} 1-\mathrm{C} 6)$ that are bonded to the protons $\mathrm{H} 1-\mathrm{H} 6$ and the oxygen atom bonded to $\mathrm{C} 4$ is denoted by $\mathrm{O} 4$. In the NMR spectrum the DTAB protons exhibited six discrete peaks: $\mathrm{H}_{\omega}, \mathrm{H}_{\lambda-\theta}, \mathrm{H}_{\gamma-\eta}, \mathrm{H}_{\beta}, \mathrm{H}_{\alpha}$, and $\mathrm{H}_{\mathrm{N}} \cdot{ }^{17)}$ 
namics simulation of the shuttling of $\alpha-\mathrm{CD}$ on the alkyl chain has been reported. ${ }^{7,8)}$

In the present research, we investigated molecular motions of $\alpha-\mathrm{CD}$ on the dodecyl chain of DTAB by molecular dynamics simulations under the conditions where no motions of DTAB and all molecular motions of $\alpha$-CD were presumed. For the $\alpha$-CD molecule, translational and rotational motions, deformation of the macrocycle, and rattling motions around the center of the dodecyl chain were analyzed. Some of these predictions were compared with the published NMR data on the DTAB- $\alpha-C D$ complex. ${ }^{17)}$ In particular, the effective distance between intermolecular protons was calculated by taking into account the translational distribution of $\alpha-\mathrm{CD}$ and were compared with the observed ROE intensities.

\section{Experimental}

Molecular Dynamics Simulations ${ }^{25)}$ Molecular dynamics simulations of the $1: 1$ complex between DTAB and $\alpha$-CD were carried out using Discover 3 Version 98 (Accelrys, Inc.) on a Silicon Graphics Octane workstation. The periodic boundary condition was applied on a $3 \times 2 \times 2-\mathrm{nm}$ solid cubic cell in which the complex was centered. The cell was soaked with 327 water molecules and contained total atoms of 1157 . The potential types of each atom were derived from the CVFF (Consistent Valence Force Field) force field. The cut off distance for van der Waals and electrostatic forces was $1.6 \mathrm{~nm}$. The potential energy of the system was minimized. The initial velocities of atoms were given by the Maxwell-Boltzmann distribution at $10 \mathrm{~K}$. The equilibration of temperature at $298.2 \mathrm{~K}$ was attained out by molecular dynamics simulations of every $1 \mathrm{fs}$ during $5000 \mathrm{fs}$ at $298.2 \mathrm{~K}$. Temperature was maintained at $298.2 \mathrm{~K}$ by the direct velocity scaling method. The equilibration of temperature and total energy was attained at $c a$. $1 \mathrm{ps}$. Then molecular dynamics simulations were performed under a constant volume and a constant density (the $N V T$ ensemble). The dynamics simulations were performed every $1 \mathrm{fs}$ during $100 \mathrm{ps}$. The data trajectory was sampled every $100 \mathrm{fs}: 1000$ structures were subject to analysis.

Molecular dynamics simulations of a free $\alpha$-CD molecule were also performed to estimate the effect of DTAB under the above-mentioned conditions.

Molecular Mechanics Calculations Molecular mechanics calculations with CVFF and Amber force fields of Discover 3 (Version 98) were carried out under the same conditions as the molecular dynamics simulations. The most populated structure of the DTAB- $\alpha$-CD complex obtained by molecular dynamics simulations was used as the initial structure and was energyoptimized to determine the final structure.

Definition of Molecular Coordinates The molecular dynamics simulations and molecular mechanics calculations were carried out on the basis of the following assumptions. All carbon atoms of the dodecyl chain of DTAB are in the trans arrangement. The DTAB molecule has no flexibility and no motions, whereas the $\alpha-\mathrm{CD}$ molecule has full flexibility and full motions.

In Fig. 2, all atoms of carbon and nitrogen of a DTAB molecule are shown in points and the lines between these atoms display chemical bonds. The shape of an $\alpha$-CD molecule is a truncated cone with a cavity. All carbon atoms of $\alpha-\mathrm{CD}$ are shown as black balls. Six oxygen atoms (O4) at position 4 of the glucose unit are shown as pink balls and form a hexagon. The other oxygen atoms are shown as red balls, respectively. All hydrogen atoms of DTAB and $\alpha$-CD are omitted for clarity. The Cartesian coordinates are defined for the DTAB molecule. All $\mathrm{C}-\mathrm{C}$ bonds of DTAB in the trans conformation were fixed on the $x y$ plane. The central line $(y=0$ and $z=0)$ of the long side of this plane was defined as the $x$-axis and the short side of this plane was defined as the $y$-axis. The nitrogen atom was located at $x=0$, $y=0.049 \mathrm{~nm}$, and $z=0$. The symmetry axis of $\alpha$-CD was placed through the $x$-axis. All atoms of $\alpha$-CD were determined in the Cartesian coordinate system as a function of time.

Two orientations of the $1: 1$ complex were considered: the terminal methyl group of DTAB is incorporated from the wide secondary hydroxyl side (secondary-in) or the narrow primary hydroxyl side (primary-in) of $\alpha$ CD. The results of the former orientation, shown in Fig. 2, will be reported, unless specially stated.

\section{Results}

Translational Motion All atoms of a DTAB molecule were fixed in all motions. All $\mathrm{C}-\mathrm{C}$ bonds of DTAB were fixed in the trans conformation on the $x y$ plane. The $x$ axis is the central line of the long side of this plane. The positions of carbon and oxygen atoms of DTAB are shown in Fig. 2, where all hydrogen atoms are omitted for clarity. An $\alpha$-CD molecule can move on the $x y$ plane with all degrees of freedom of motions. The center of the $\alpha$-CD molecule can move translationally along the $x$-axis. This translational motion of $\alpha$-CD was expressed by the position averaged over the $x$-coordinates for $6 \mathrm{O} 4$ atoms that are shown by pink balls in Fig. 2.

As shown in Fig. 3, the $\alpha$-CD molecule rapidly shuttled on the dodecyl chain of DTAB. The starting and returning positions depend irregularly on each shuttling motion. From these data the probability (population) in which the average $\mathrm{O} 4$ atom is present near one of the methylene carbon atoms of DTAB was calculated, and is shown in Panel a of Fig. 4. The average $\mathrm{O} 4$ atom has the maximum probability between the carbon atoms of C6 $(x=0.754 \mathrm{~nm})$ and $\mathrm{C} 7(x=0.882 \mathrm{~nm})$ of DTAB.

Molecular dynamics simulations were also carried out for the DTAB- $\alpha$-CD complex for the primary-in structure. The average $\mathrm{O} 4$ plane for this complex is shown as a function of the position of the carbon atom of DTAB in Panel b of Fig. 4. The average $\mathrm{O} 4$ atom has the maximum probability between

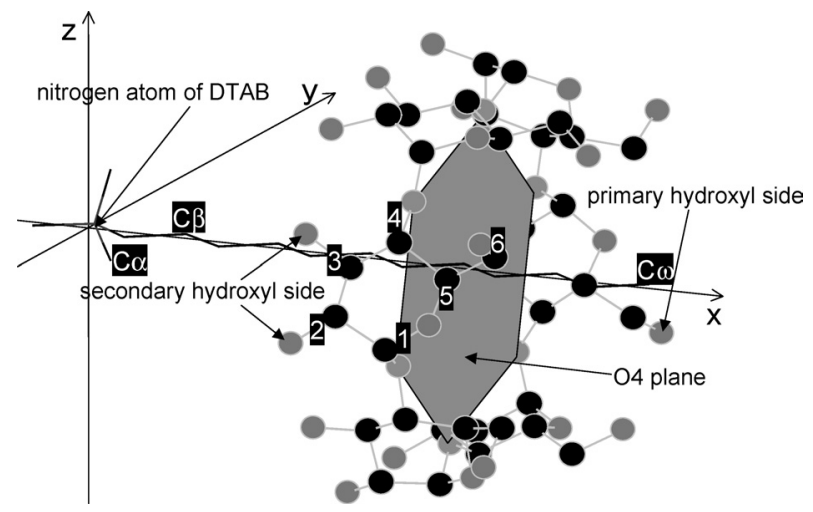

Fig. 2. Definition of Cartesian Coordinates of a Secondary-in Complex between DTAB and $\alpha-\mathrm{CD}$

The nitrogen atom of DTAB is located at $x=0, y=0.049 \mathrm{~nm}$, and $z=0$. The 12 carbon atoms of the dodecyl chain in the trans conformation are fixed on the $x y$ plane. All carbon and nitrogen atoms are shown as points and the chemical bonds among them are shown as lines. All hydrogen atoms of DTAB are omitted for clarity. A flexible $\alpha$-CD molecule can move through the DTAB molecule. Six O4 atoms of $\alpha$-CD form a hexagon.

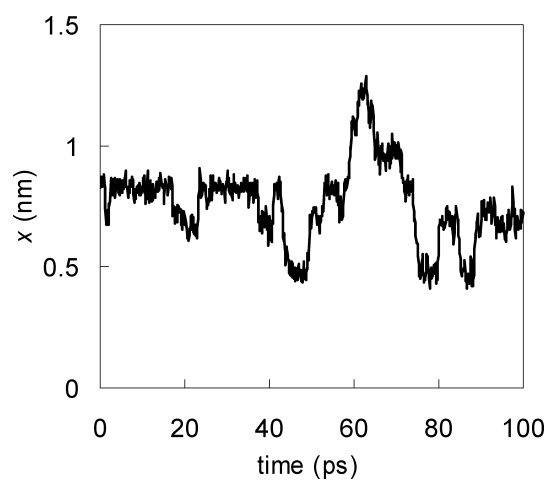

Fig. 3. Translational Motion of $\alpha$-CD

The $x$-coordinate of the $\mathrm{O} 4$ plane of $\alpha$-CD is shown every $100 \mathrm{fs}$. 


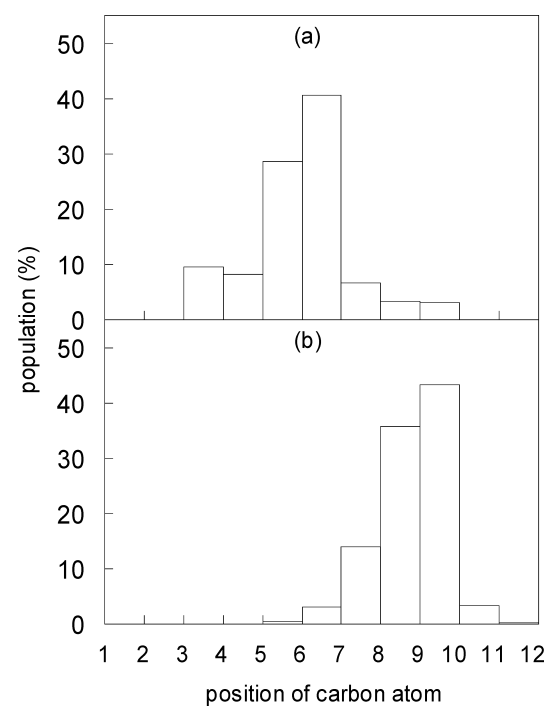

Fig. 4. Distributions of the $x$-Coordinate of the O4 Plane in Cases Where the Methyl Group Is Incorporated from (a) the Secondary Hydroxyl Side and (b) the Primary Hydroxyl Side

The ordinate denotes the position of the carbon atom of a dodecyl chain.

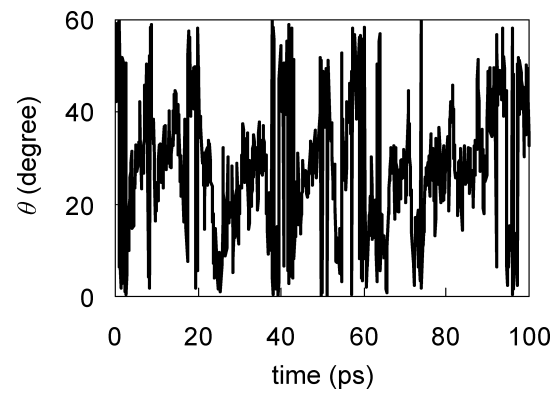

Fig. 5. Rotational Angle of $\alpha$-CD around the Dodecyl Chain Every $100 \mathrm{fs}$

the carbon atoms of $\mathrm{C} 9$ and $\mathrm{C} 10$ of DTAB.

Rotational Motion Six $\mathrm{O} 4$ atoms of the $\alpha$-CD molecule form a nearly normal hexagon. The angle between the $x y$ plane and the plane vertical to this hexagon and passing through the diagonal line of this hexagon was defined as $\theta$. This rotational angle changed with elapsed time: the $\alpha$-CD molecule rotates around the $x$-axis. As shown in Fig. 5, the $\alpha$-CD molecule exhibited a rapid restricted rotation around $\theta=30^{\circ}$. The starting and returning angles depend irregularly on each rotation. From these data the rotational-angle distribution was calculated at intervals of $5^{\circ}$, and is shown as a function of $\theta$ in Fig. 6. The distribution is rather wide, centered at $\theta=27.5^{\circ}$. Figure 7 shows the most probable structure of the complex projected on the $y z$-plane. The most probable angle was also $\theta=27.5^{\circ}$ for the primary-in complex (data not shown).

Deformation of Macrocycle The macrocycle of $\alpha$-CD is significantly distorted in crystals of $\alpha-\mathrm{CD} \cdot 6 \mathrm{H}_{2} \mathrm{O} \cdot{ }^{28)}$ Three successive $\mathrm{O} 4$ atoms for a normal hexagon form an angle of $120^{\circ}$, because most of the $6 \mathrm{O} 4$ atoms are on a plane. The deviation, $\Delta \phi$, from this angle was regarded as a measure of deformation of the macrocycle. The root, $\Delta \phi_{\mathrm{av}}$, of the average of $(\Delta \phi)^{2}$ over 6 angles per macrocycle was used as the degree of deformation of the macrocycle. The distribution of $\Delta \phi_{\mathrm{av}}$ in intervals of $0.5^{\circ}$ was calculated in the presence and absence of DTAB in water. As shown in Panel b of Fig. 8, the

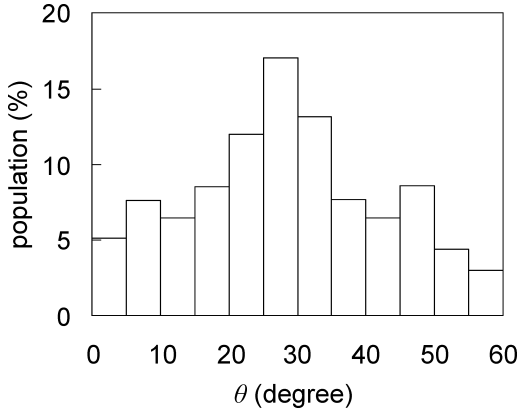

Fig. 6. Distributions of Rotational Angles in Intervals of 5 Degrees

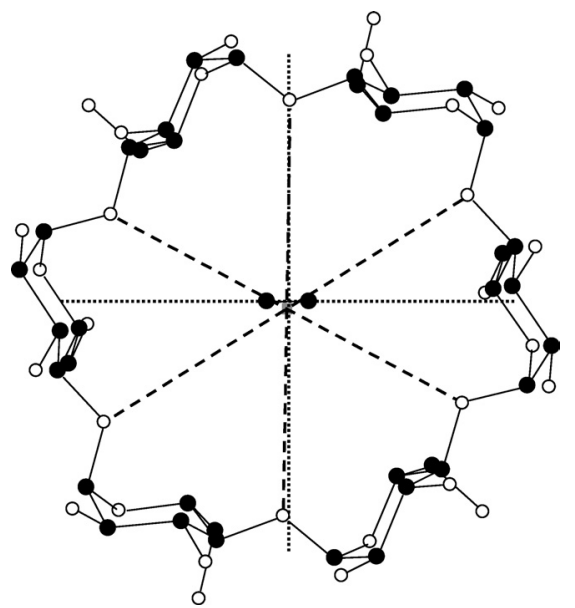

Fig. 7. The Most Probable Stick-and-Ball Structure $\left(\theta=27.5^{\circ}\right)$ of the DTAB and $\alpha$-CD Complex Projected on the $y z$ Plane

Here, the carbon and oxygen atoms of $\alpha$-CD are shown by closed and open circles, respectively, DTAB is shown by two closed circles near the center, and the $N$-trimethyl group of DTAB and all hydrogen atoms are omitted for clarity. The square shows the most probable position $(\rho=0.025 \mathrm{~nm})$ of the center of $\alpha-\mathrm{CD}$. The three diagonal lines between $6 \mathrm{O} 4$ atoms are shown by dashed lines.

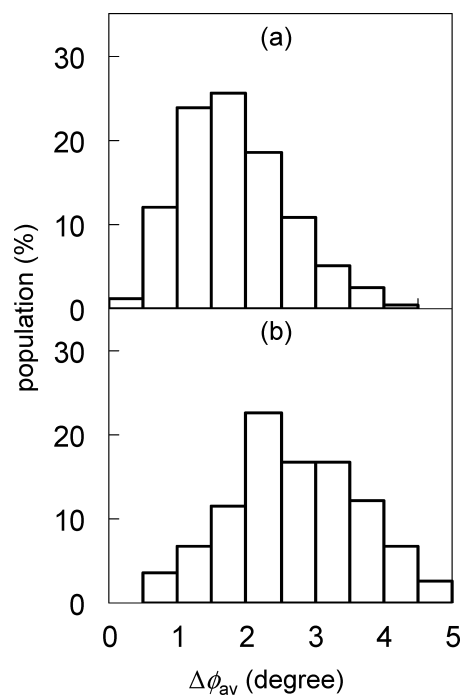

Fig. 8. Distributions of Distortion of a Hexagon Formed by 6 O4 Atoms from the Normal Hexagon at Intervals of $\phi=0.5^{\circ}$ in (a) the Presence and (b) Absence of DTAB

most probable value of $\Delta \phi_{\mathrm{av}}$ in the absence of DTAB is $2.3^{\circ}$, whereas it is decreased to $1.7^{\circ}$ in the presence of DTAB (Panel a). The $\Delta \phi_{\text {av }}$ values for the primary-in complex (data 


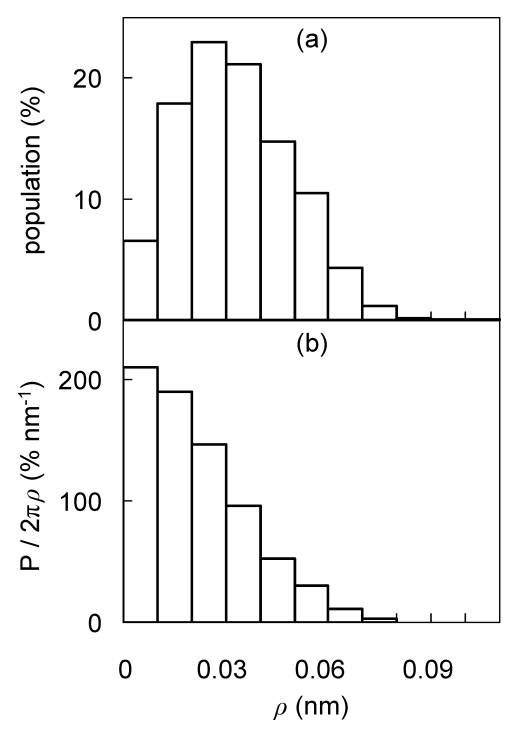

Fig. 9. (a) Radial Deviation of the Center of $\alpha-\mathrm{CD}$ from the Center of the Dodecyl Carbon Chain of DTAB and (b) Its Density

not shown) are the same as those for the secondary-in complex.

Displacement between the Centers of $\alpha-C D$ and DTAB The central line of the carbon chain of DTAB is defined at $y=0$ and $z=0$. The $y$ and $z$ coordinates at the center of a hexagon formed by $6 \mathrm{O} 4$ atoms were calculated, and then the deviation $\rho$ of this center from the center of the DTAB molecule was calculated from $\left(y^{2}+z^{2}\right)^{1 / 2}$. We can regard $\rho$ as a measure of rattling motion of $\alpha-\mathrm{CD}$ around the center of the carbon chain. The distribution of $\rho$ is shown in Panel a of Fig. 9. The most probable deviation is $\rho=0.025 \mathrm{~nm}$. Because the area of a circle having a $\rho$ value is proportional to $2 \pi \rho$ in the cylindrical polar coordinate system, the density of population must be divided by $2 \pi \rho$. As shown in Panel $b$ of Fig. 9 , the density of population has a maximum at the center of the dodecyl chain $(y=0$ and $z=0)$, as expected. The dodecyl chain is on the $x y$ plane and the O4 plane is parallel to the $y z$ plane. Therefore, the distribution of $\rho$ depended slightly on the sign of $y$ or $z$, as shown in Fig. 7. The distribution of the $\rho$ values for the primary-in complex (data not shown) is the same as those for the secondary-in complex.

Comparison of Predicted Structures with NMR Data Figure 10 shows five structures of the DTAB- $\alpha-C D$ complex. Panels a and b in Fig. 10 are the most populated molecular dynamics structures of the secondary-in and primary-in complexes (Fig. 4). Further, these complexes were energyoptimized by molecular mechanics with CVFF and Amber force fields. The CVFF and Amber structures of the secondary-in complex are shown in Panels c and d of Fig. 10. As is given in Table 1, the total potential energy for the secondaryin complex is smaller than the primary-in complex for both of the force fields. This is consistent with the NMR structure (Panel e in Fig. 10). The largest contributions to the difference between the two structures for the CVFF and Amber force fields are the electrostatic and van der Waals repulsive energies, respectively. The characteristic structural parameters for the five structures are given in Table 2.

The most probable structure of the $1: 1$ DTAB- $\alpha-C D$ complex had been estimated from volumes (intensities) of in-

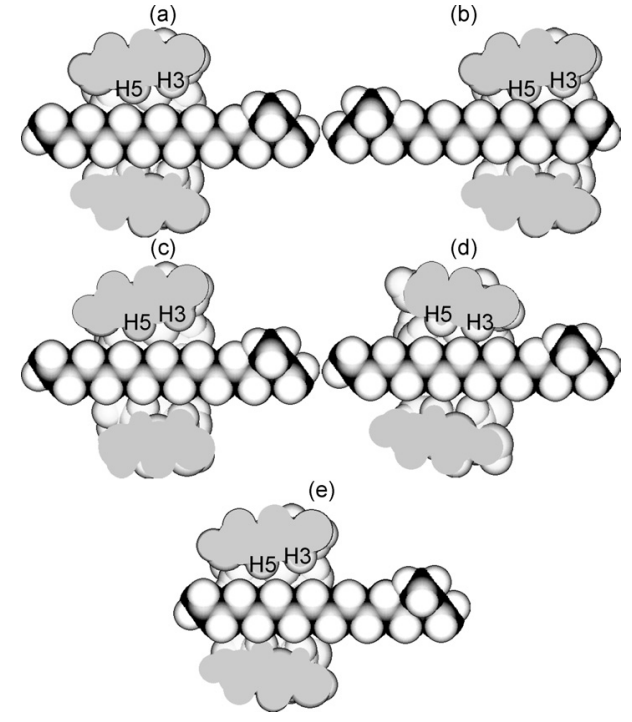

Fig. 10. The Most Populated Molecular Dynamics Structures for (a) the Secondary-in Complex and (b) the Primary-in Complex, (c) the CVFF Molecular Mechanics Structure, (d) the Amber Molecular Mechanics Structure, and (e) the NMR Structure for the Secondary-in Complex between DTAB and $\alpha-\mathrm{CD}$

Table 1. Energies $(\mathrm{kcal} / \mathrm{mol})$ for the Secondary-in Complex (Structures c and $\mathrm{d}$ in Fig. 10) and the Primary-in Complex Calculated by Molecular Mechanics with CVFF and Amber Force Fields

\begin{tabular}{|c|c|c|c|c|}
\hline \multirow{2}{*}{ Energy } & \multicolumn{2}{|c|}{ CVFF } & \multicolumn{2}{|c|}{ Amber } \\
\hline & Secondary-in & Primary-in & Secondary-in & Primary-in \\
\hline Bond & 142.5 & 142.5 & 132.1 & 135.5 \\
\hline Angle & 137.2 & 138.3 & 73.4 & 71.9 \\
\hline Torsion & 16.0 & 17.2 & 34.8 & 35.4 \\
\hline $\begin{array}{l}\text { van der Waals } \\
\text { repulsive }\end{array}$ & 2206.2 & 2197.4 & 2042.8 & 2097.3 \\
\hline $\begin{array}{c}\text { van der Waals } \\
\text { dispersive }\end{array}$ & -1504.6 & -1501.1 & -1358.0 & -1369.2 \\
\hline Electrostatic & -4864.1 & -4839.0 & -5684.0 & -5713.7 \\
\hline Hydrogen-bond & 0.0 & 0.0 & -7.3 & -5.9 \\
\hline Total potential & -3866.9 & -3844.7 & -4766.2 & -4748.7 \\
\hline
\end{tabular}

Table 2. Comparison among the Most Populated Molecular Dynamics Structure, the Average Molecular Dynamics Structure, and the Molecular Mechanics Structures Calculated with CVFF and Amber Force Fields for the Secondary-in Complex

\begin{tabular}{lccccc}
\hline \hline & $\begin{array}{c}x \\
(\mathrm{~nm})\end{array}$ & $\begin{array}{c}\theta \\
(\text { degree })\end{array}$ & $\begin{array}{c}\Delta \phi_{\mathrm{av}} \\
(\text { degree })\end{array}$ & $\begin{array}{c}\rho \\
(\mathrm{nm})\end{array}$ & $R$ \\
\hline $\begin{array}{c}\text { Most populated } \\
\text { MD structure }\end{array}$ & 0.818 & 27.5 & 1.75 & 0.025 & 0.695 \\
$\begin{array}{c}\text { Average MD } \\
\text { structure }\end{array}$ & $-a)$ & $-a)$ & $-a)$ & $-{ }^{a)}$ & 0.599 \\
$\begin{array}{c}\text { CVFF structure } \\
\text { Amber structure }\end{array}$ & 0.759 & 14.5 & 3.15 & 0.010 & 0.554 \\
NMR structure & 0.789 & 12.0 & $\begin{array}{c}1.63 \\
{ }^{a} \text { ) }\end{array}$ & 0.035 & 0.619 \\
\hline
\end{tabular}

a) Not calculated, because of required long time and few merits.

termolecular cross-peaks in the NMR ROESY spectrum. ${ }^{17}$ This NMR structure was determined using the following equation with the empirical parameters $k$ and $a$ :

$\mathrm{ROE} / n_{\mathrm{CD}} n_{\mathrm{DT}}=k d_{\mathrm{eff}}^{-a}$ 


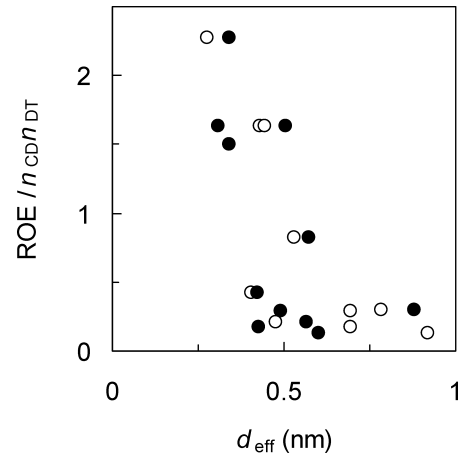

Fig. 11. Correlations between the Observed ROE Intensity and the Effective Interproton Distance of the Secondary Side-in Complex for the Most Populated Molecular Dynamics Structure (Open Circles) and the Average Molecular Dynamics Structure (Closed Circles)

The ROE data on the cross-peaks of the protons H3, H5, and $\mathrm{H} 6$ with the DTAB protons are taken from ref. 17, although those data on the protons $\mathrm{H} 2$ and $\mathrm{H} 4$ were omitted therefrom. ${ }^{18)}$

Here, ROE stands for the ROE intensity for an intermolecular cross-peak and $n_{\mathrm{CD}}$ and $n_{\mathrm{DT}}$ denote the numbers of equivalent protons for $\alpha$-CD and DTAB that were assigned to this cross-peak. The effective distance, $d_{\mathrm{eff}}$, is defined as ${ }^{17,26,27)}$ :

$$
\left(d_{\mathrm{eff}}\right)^{-6}=\left(1 / n_{\mathrm{CD}} n_{\mathrm{DT}}\right) \sum_{i=1}^{n_{\mathrm{CD}}} \sum_{j=1}^{n_{\mathrm{DT}}} d_{\mathrm{CD}_{\mathrm{D}} \mathrm{DT}}^{-6}
$$

Here, $d_{\mathrm{CD}_{i} \mathrm{DT}_{i}}$ denotes the distance between proton $\mathrm{CD}_{i}$ of $\alpha-$ $\mathrm{CD}$ and proton $\mathrm{DT}_{j}$ of DTAB. From Eqs. 1 and 2 we can expect that $\mathrm{ROE} / n_{\mathrm{CD}} n_{\mathrm{DT}}$ increases, as two protons become closer to each other. We assumed that the best NMR structure has the largest correlation coefficient in Eq. $1 .{ }^{17)}$

In the ROESY spectrum of the $1: 1$ DTAB- $\alpha$-CD complex, DTAB had six discrete peaks ( $N$-methyls, $\alpha$-methylene, $\beta$-methylene, $\gamma$ - $\eta$-methylenes, $\lambda$ - $\theta$-methylenes, and terminal $\omega$-methyl) and $\alpha$-CD had five discrete peaks (H1, H2,4, H3, $\mathrm{H} 5, \mathrm{H} 6)$. The protons $\mathrm{H}_{\gamma-\eta}$ and $\mathrm{H}_{\lambda-\theta}$ are bonded with the carbon atoms $\mathrm{C} 3-\mathrm{C} 7$ and $\mathrm{C} 8-\mathrm{C} 11$ of DTAB, respectively (Fig. 1). Because the protons $\mathrm{H} 3, \mathrm{H} 5$, and $\mathrm{H} 6$ are located inside the $\alpha$-CD cavity, they had larger cross-peaks than the protons $\mathrm{H} 1, \mathrm{H} 2$, and $\mathrm{H} 4$ outside the cavity. ${ }^{17,18)}$ The signals of the protons $\mathrm{H} 6 S$ and $\mathrm{H} 6 \mathrm{R}$ of $\alpha$-CD were overlapped with each other and were regarded as a single signal of the protons H6. This signal $\left(n_{\mathrm{CD}}=12\right)$ had three cross-peaks with the signals of the protons $\mathrm{H}_{\gamma-\eta}\left(n_{\mathrm{DT}}=10\right), \mathrm{H}_{\lambda-\theta}\left(n_{\mathrm{DT}}=8\right)$, and $\mathrm{H}_{\omega}$ $\left(n_{\mathrm{DT}}=3\right)$ of DTAB. Each of the signals of $\mathrm{H} 3$ and $\mathrm{H} 5$ of $\alpha$ CD had cross-peaks with signals of $\mathrm{H}_{\beta}, \mathrm{H}_{\gamma-\eta}, \mathrm{H}_{\lambda-\theta}$, and $\mathrm{H}_{\omega}$ of DTAB. The effective distances between these intermolecular protons were calculated for several structures of the DTAB and $\alpha$-CD complex and were plotted against the observed ROE intensities in Fig. 11. First, the effective distances were calculated on the basis of the most populated molecular dynamics structure of the secondary-in complex and are shown by the open circles of Fig. 11. From the plots the best fit values of $k$ and $a$ in Eq. 1 and the correlation coefficient $R$ were evaluated. The $R$-value is given in Table 2 .

However, it was found that an $\alpha$-CD molecule shuttles on the DTAB molecule. Then we must take into consideration this translational motion to calculate the effective distances. A time-average effective distance can be calculated from:

$$
\left(d_{\mathrm{eff}}\right)^{-6}=\left(1 / n_{\mathrm{CD}} n_{\mathrm{DT}}\right) \sum_{k=1}^{n_{\mathrm{CD}}} P_{k} \sum_{i=1}^{n_{\mathrm{DT}}} \sum_{j=1} d_{\mathrm{CD}_{\mathrm{i}} \mathrm{DT}_{j}}^{-6}
$$

Here $P_{k}$ denotes the population for a structure of the complex and is shown in Panel a of Fig. 4. A correlation coefficient of 0.599 was obtained for this average molecular dynamics structure. This is worse than the most populated structure (Panel a of Fig. 10). The correlation coefficients for the CVFF and Amber structures (Panels c and d of Fig. 10) were also calculated and are given in Table 2 .

The most important factor in these correlations is the position $x$ of the $\mathrm{O} 4$ plane. The effective distances at $x=0.981 \mathrm{~nm}$ were calculated as a function of the rotational angle $\theta$ and were plotted against the ROE intensities. The $R$-value changed between 0.812 and 0.821 (data not shown). Thus, a slightly better NMR structure of the DTAB- $\alpha$-CD complex was obtained. The radial deviation $\rho$ was a more minor factor: the correlation coefficient for the structure at $\rho=0.025$ $\mathrm{nm}$ was better than that at $\rho=0$ by 0.002 .

\section{Discussion}

To our knowledge, this is the first report on a molecular dynamics study of surfactant $-\mathrm{CD}$ complexes. The DTAB- $\alpha$ $\mathrm{CD}$ complex is a good system to investigate shuttling by molecular dynamics. To simplify data analysis, we assumed that DTAB is a rigid and fully extended molecule. This is a reasonable assumption, because it is the most probable conformation of a DTAB molecule. No decomplexation between DTAB and $\alpha-\mathrm{CD}$ occurred during our molecular dynamics simulations: the binding constant for the DTAB and $\alpha-\mathrm{CD}$ system is infinite. The observed binding constant is 18200 $\mathrm{M}^{-1}$, a large value. ${ }^{17)}$

The most important finding of the present research is that the NMR structure of the $1: 1$ DTAB- $\alpha$-CD complex is rather close to the structures estimated by molecular mechanics and dynamics calculations, in particular very close to the most populated molecular dynamics structure (Fig. 10). In Table 2 it is noted that the most populated molecular dynamics structure (Panel a) is better than the molecular mechanics structures (Panels $\mathrm{c}$ and $\mathrm{d}$ ). The solution structure changes with time. However, the NMR data provide only the time-average structure. Furthermore, the ROE intensity of a crosspeak is influenced by a few factors other than the interproton distance. ${ }^{17,18,26,27)}$ Although the NOE intensity, instead of the ROE intensity, can exclude these complicating factors, it is small for molecules of middle size, such as cyclodextrins and their complexes. ${ }^{18,26,27)}$ In both theory and experiment, we assumed that a DTAB molecule adopts the fully extended conformation. Account of other folded structures would yield better structures of the DTAB $-\alpha-\mathrm{CD}$ complex. Although we employed the CVFF forcefield for molecular dynamics simulations, other force fields may yield better predictions. Irrespective of these uncertainties, the present agreement between theory and experiment is satisfactory.

The correlation between the NMR ROE intensity and the interproton distance for the $1: 1 \mathrm{DTAB}-\alpha-\mathrm{CD}$ complex is worse than those of $\alpha$-CD complexes with shorter surfactants. This finding was interpreted as showing the shuttling of $\alpha$-CD. ${ }^{17)}$ This interpretation was quantitatively confirmed on the basis of the present molecular dynamics simulations. The other NMR evidence for shuttling was based on the 
chemical shift variations of DTAB protons in the $\alpha-\mathrm{CD}$ cavity. ${ }^{17)}$ To confirm this suggestion quantitatively, we need the numerical relation between the chemical shift variation and the position of a proton for a non-shuttling surfactant. However, we could not find such a surfactant. Propanol and propanesulfonate are good candidates, but these molecules are too small to cover the entire region of the $\alpha$-CD cavity. ${ }^{24}$ The hexyl chain can cover this region, but it would shuttle slightly inside the cavity. ${ }^{16)}$ Therefore, we did not attempt to analyze the chemical shift data of DTAB quantitatively.

It has been demonstrated by a scanning tunneling electron microscopy, chemistry, and molecular dynamics simulations that $\alpha$-CD molecules shuttle on a polyethylene oxide chain. ${ }^{29-32)}$ The present molecular dynamics study predicts that an $\alpha$-CD molecule shuttles on a hydrocarbon chain. Very recently, cyclodextrin-based interlocked molecules attract strong research interests. ${ }^{31)}$ The DTAB- $\alpha-\mathrm{CD}$ complex is an interlocked molecule called a pseudorotaxane. The shuttling process in [2] rotaxanes was studied by molecular dynamics simulations. ${ }^{33)}$

A molecular rotation of $\alpha-\mathrm{CD}$ will be rather insensitive to NMR data on the DTAB- $\alpha$-CD system. A fast rotation gives a spectrum averaged over different rotational angles. The NMR spectrum of the DTAB- $\alpha$-CD system at $298.2 \mathrm{~K}$ indicates a rapid rotation. ${ }^{17)}$ At lower temperature a slower rotation will be observed by NMR. A slower molecular rotation results in more nonequivalent signals in NMR spectra, so that the speed of rotation may be determined. ${ }^{34)}$ The correlation between the observed ROESY intensity and the rotational angle for the DTAB- $\alpha$-CD complex had not yet been investigated. ${ }^{17)}$ In the present research we have determined a slightly better structure at a rotational angle of $0^{\circ}$ (Table 2). This angle is close to $\theta=3^{\circ}$ for the propanesulfonate $-\alpha$-CD complex in crystals, ${ }^{35)}$ although it is different from the values (Table 2) predicted by molecular dynamics and molecular mechanics. Because the chemical shifts of the $\alpha$-CD protons for the benzenesulfonate- $\alpha$-CD system depend on the rotational angle, we estimated a rotational angle of $\theta=30^{\circ} .{ }^{36)}$

The displacement of $\alpha$-CD from the center of the dodecyl chain will be rather insensitive to NMR data on the DTAB $-\alpha-\mathrm{CD}$ system. A distortion of the $\alpha-\mathrm{CD}$ macrocycle in solution will be difficult to be detected by most experimental measurements. In crystals the $\alpha$-CD macrocycle is significantly distorted, ${ }^{28}$ and complex formation decreases this distortion. ${ }^{4,5,8,36)}$ From vicinal spin-spin coupling constants of $\beta$-CD protons, we had suggested a large deformation of the $\beta$-CD macrocycle induced by diheptanoyllecithin. ${ }^{23)}$

Although a great many of time-dependent data on the DTAB $-\alpha-C D$ complex were obtained by the present simulations, none of them were analyzed because of deficiency of experimental data to be compared with. From NMR data, it was suggested that $\alpha$-CD on the DTAB molecule shuttles fast on the ${ }^{1} \mathrm{H}-\mathrm{NMR}$ time scale. ${ }^{17)}$ This finding is consistent with the present simulations. Ultrafast guest dynamics in cyclodextrin nanocavities was recently reviewed and may be analyzed by molecular dynamics simulations. ${ }^{37)}$

Molecular dynamics simulations provide a starting place for testing rationales and drawing new conclusions. Like any other good experimental techniques, molecular modeling can often raise more questions than it answers. A detailed and through theoretical understanding at the molecular level may lead to the rational design of cyclodextrin-based novel materials with tailored properties, such as media for chromatography and useful catalysts for organic reactions. Because computational chemistry can help rationalize experimental observation, provide information not amenable to experimentation, and even make predictions concerning the outcome of future experiments, it will become more widely accepted by experimental scientists as a valuable adjunct to their experimental studies. $^{7,8)}$

Acknowledgements Thanks are due to Mr. Daisuke Shimamoto and Mr. Yutaka Yamashita for some analysis of molecular dynamics data. The present research was supported by a Grant-in-Aid for the Frontier Research Program from the Ministry of Education, Science, Sports, Culture, and Technology of Japan, which is gratefully acknowledged.

\section{References and Notes}

1) Saenger W., Angew. Chem. Int. Ed., 19, 344-362 (1980).

2) Szejtli J., "Cyclodextrin Technology," Chap. 2 and 3, Kluwer Academic Publishers, Dordrecht, The Netherlands, 1988.

3) Fenyvesi É., Szente L., Russell N. R., McNamara M., "Cyclodextrins," Chap. 10, ed. by Szejtli J., Osa T., Pergamon, London, 1996.

4) Harata K., Chem. Rev., 98, 1803-1827 (1998).

5) Saenger W., Jacob J., Gesseler K., Steiner T., Hoffmann D., Sanbe H., Koizumi K., Smith S., Takaha T., Chem. Rev., 98, 1787-1802 (1998).

6) Schneider H.-J., Hacket F., Rüdiger V., Ikeda H., Chem. Rev., 98, 1755-1875 (1998).

7) Sherrod M. J., "Spectroscopic and Computational Studies of Supramolecular Systems," Chap. 7, ed. by Davies J. E. D., Kluwer Academic Publishers, Dordrecht, The Netherlands, 1992.

8) Lipkowitz K. B., Chem. Rev., 98, 1829-1873 (1998).

9) Wan Yunus W. M. Z., Taylor J., Bloor D. M., Hall D. G., Wyn-Jones E., J. Phys. Chem., 96, 8979-8982 (1992).

10) Funasaki N., Neya S., Langmuir, 16, 5343-5346 (2000).

11) Funasaki N., Yodo H., Hada S., Neya S., Bull. Chem. Soc. Jpn., 65, 1323-1330 (1992).

12) Funasaki N., Ohigashi M., Hada S., Neya S., Langmuir, 16, 383-388 (2000).

13) Wilson L. D., Verrall R. E., Can. J. Chem., 76, 25-34 (1998).

14) Ishikawa S., Neya S., Funasaki N., J. Phys. Chem., B, 102, 25022510 (1998).

15) Avram L., Cohen Y., J. Org. Chem., 67, 2639-2644 (2002).

16) Funasaki N., Ishikawa S., Neya S., J. Phys. Chem., B, 107, 10094 10099 (2003)

17) Funasaki N., Ishikawa S., Neya S., J. Phys. Chem., B, 108, $9593-$ 9598 (2004). Therein the ROE cross-peaks of the protons H2 and 4 with the DTAB protons were employed to determine the structures of the $1: 1$ complex of DTAB and $\alpha$-CD. These, however, will be false peaks that are relayed by vicinal spin-spin couplings of the protons $\mathrm{H} 2$ and $\mathrm{H} 4$ with the protons $\mathrm{H} 3$ and $\mathrm{H} 5{ }^{18)}$ This difference in the ROE data employed little affected the NMR structure.

18) Claridge T. D. W., "High-Resolution NMR Techniques in Organic Chemistry," Chap. 8, Pergamon, London, 1999.

19) Rontoyianni A., Mavridis I. M., Acta Crystallogr., C, 52, 2277-2281 (1996).

20) Rontoyianni A., Mavridis I. M., Supramolec. Chem., 10, 213-218 (1999).

21) Gonźalez-Gaitano G., Crespo A., Compostizo A., Tardajos G., J. Phys. Chem., B, 101, 4413-4421 (1997)

22) Ishikawa S., Hada S., Neya S., Funasaki N., J. Phys. Chem., B, 103, 1208 - 1215 (1999).

23) Funasaki N., Ishikawa S., Neya S., Langmuir, 18, 1786-1790 (2002).

24) Funasaki N., Ishikawa S., Neya S., J. Phys. Chem., B, 106, 64316436 (2002).

25) Haile J. M., "Molecular Dynamics Simulation," Chap. 2 and 6, John Wiley, New York, 1992

26) Kessler H., Seip S., "Two-dimensional NMR Spectroscopy," 2nd ed., Chap. 5, ed. by Croasmun W. R., Carlson R. M. K., Wiley-VCH, New York, 1994.

27) Neuhaus D., Williamson M. P., "The Nuclear Overhauser Effect in Structural and Conformational Analysis," 2nd ed., Chap. 9 and 12, 
Wiley-VCH, New York, 2000.

28) Saenger W., Noltemeyer M., Manor P. C., Hingerty B., Klar B., Bioorg. Chem., 5, 187-195 (1976).

29) Shigekawa H., Miyake K., Sumaoka J., Harada A., Komiyama M., J. Am. Chem. Soc., 122, 5411-5412 (2000).

30) Harada A., Acc. Chem. Res., 34, 456- 464 (2001).

31) Nepogodiev S., Stoddart J. F., Chem. Rev., 98, 1959-1976 (1998).

32) Pozuelo J., Mendicuti F., Mattice W. L., Macromolecules, 30, 36853690 (1997).
33) Grabuleda X., Ivanov P., Jaime C., J. Phys. Chem., B, 107, 75827588 (2003).

34) Neya S., Funasaki N., Nakamura M., Biochim. Biophys. Acta, 1117, 243-250 (1992)

35) Harata K., Bull. Chem. Soc. Jpn., 50, 1259-1266 (1977).

36) Funasaki N., Yamaguchi H., Ishikawa S., Neya S., J. Phys. Chem., B, 105, 760-765 (2001).

37) Douhal A., Chem. Rev., 104, 1955-1976 (2004). 